\title{
CONTROL STRATEGY OF THE INPUT STREAM ON THE ONLINE CHARGING SYSTEM IN PEAK OF LOAD Control Strategy of the input stream on the server
}

charge in peak load

Globa Larisa, Skulysh Mariia, Reverchuk Andriy

SitronicsTS CZ a.s.

Institute of Telecommunication system

National Technical University of Ukraine "Kiev Polytechnic Institute", Kiev, Ukraine

тел.: +380-44-4068209, e-mail: mb s@ukr.net

Abstract - The report presents a strategy for managing incoming flow, which takes into account the resource requirements for service requests for billing, taking into consideration the statistical information about the duration of the operations that will prevent the simultaneous presence in the system service requests requiring a large number of resources simultaneously

\section{СТРАТЕГИЯ УПРАВЛЕНИЯ ВХОДЯЩИМ В ПОТОКОМ НА СЕРВЕРЕ ТАРИФИКАЦИИ В МОМЕНТЫ ПИКОВИХ НАГРУЗОК}

\author{
Глоба Л.С. Скулиш М.А. Реверчук А. В
}

SitronicsTS CZ a.s.

\author{
Институт телекоммуникационных систем \\ Национальный технический институт Украины «Киевский политехнический институт» \\ Киев, Украина \\ тел.: +380-44-4068209, e-mail: $\underline{\text { mb s@ukr.net }}$
}

\begin{abstract}
- В докладе представлена стратегия управления входящим потоком, которая учитывает требования к ресурсам для обслуживания заявок на тарификацию, при этом учитывается статистическая информация о продолжительности выполнения операций, что позволит не допустить одновременного нахождения в системе заявок на обслуживание требующих одновременно большого количества ресурсов
\end{abstract}

\section{І. Вступление}

Предоставление качественных услуг операторами связи напрямую зависит от различных факторов: от скорости установления соединения, от скорости передачи информации, от надежности каналов передачи, от надежности поставщиков услуг (сервис-провайдеров). Скорость установления соединения, или значение задержки перед началом предоставления услуги, напрямую зависит от скорости обслуживания заявки на услугу на сервере тарификации. Каждая заявка на предоставление услуги обслуживается на сервере тарификации оператора связи.

Время выполнения операций ограничен, в случае его превышения заявка считается утраченной, и абонент получает сообщение о невозможности получения услуги. В такой ситуации оператор несет убытки, а в случае систематических отказов в предоставлении услуг портится репутация компании. Поэтому важно подобрать схему управления входящим потоком заявок таким образом, чтобы уменьшить вероятность превышения допустимого времени обслуживания абонентских заявок.

\section{II. Основная часть}

Процесс обслуживания абонентских заявок на сервере тарификации включает в себя последовательность стандартных операций:

- Извлечение информации об абоненте и его месте расположения,

- Извлечение информации про состояние звонка (для услуг с резервированием средств звонки, Интернет, MMS)/

- Расчет стоимости услуги на основе тарифного плана,

- Резервирование суммы или окончательный расчет

- Создание CDR (Call Detail Record)

- Отправка сообщения абоненту , если предусмотрено услугой 
В зависимости от типа услуг ресурсы необходимые для выполнения операций различны. Будем говорить про ресурсы .

Постановка задачи. Сервер мобильной связи обслуживает $\mathrm{m}$ типов сервисов. Заявкой будем называть запрос, который посылает клиент на сервер тарификации для обслуживание одного из $\mathrm{m}$ типов сервисов. Заявки одного типа сервиса обслуживаются по одинаковой схеме. Для каждого типа сервиса разработана схема обслуживания Схему обслуживания заявки можно представить как последовательный переод между $n$ функциональными блоками (ФБ).

Успешное прохождение всех ФБ в заданной последовательности обеспечивает успешное обслуживание заявки на сервере. Время обслуживания заявки на сервере ограничено, поэтому, если заявка находится в системе дольше заданного времени , то она снимается с обслуживания, а клиенту сообщается , что сеть занята.

Входными данными в задаче управления потоком заявок, поступающих на обслуживание на сервер тарификации являются:

1. Информация об объеме ресурса, необходимого для выполнения операции, предусмотренных функциональным блоком для обслуживания заявки заданного типа сервиса.

2. Иноормация о продолжительности использования ресурсов при обслуживании заявки заданного типа сервиса в каждом функциональном блоке.

3. Статистическая информация о продолжительности обслуживания заявки заданного типа сервиса в каждом функциональном блоке

Параметры сервера тарификации, как правило рассчитаны для средних значений параметров входного потока, однако в системе имеются пиковые значения количества заявок, поступивших одновременно.

Под всплеском нагрузки входного потока понимаем одновременное поступление такого количества заявок, которое больше допустимого значения.

Управление процессом обработки заявок с целью предотвращения десицита ресурсов в системе тарификации предлагается использование следующей стратегии :

- Контроль за пиками входной нагрузки: недопущение двух и более всплесков нагрузки входного потока не обслуживались одновременно в фрункциональных блоках, обслуживание в которых требует значительного количества ресурсов.

- Для этого предлагается задержать часть заявок, поступление которых совпало со всплеском нагрузки, при этом время задержки определяется так, чтобы задержанные заявки не поступали в систему до тех пор, пока предыдущий всплеск нагрузки не будет успешно обслужен в ресурсозатратном функциональном блоке.

ФБ, в которых постоянно возникают задержки из-за недостатка ресурсов сервера (оперативной памяти, дисковой памяти, процессорное время) при обработке определенных типов заявок, требующих значительных объемов вычислений по алгоритмам , положенными в основу программного обеспечения сервисов , будем называть ресурсозатратным ФБ .

$$
\text { Определение ресурсозатратного ФБ }
$$

выбранного типа сервиса осуществляется на основе информации об объеме ресурса, необходимого для выполнения операций, предусмотренных ФБ для обслуживания заявки заданного типа сервиса.

Для каждого ресурса системы, для каждого типа сервиса находятся функциональные блоки, где обслуживание является ресурсозатратным.

Проводится анализ статистической информации о длительности обслуживания заявки заданного типа сервиса в каждом функциональном блоке. Рассчитывается среднее время обслуживания заявки выбранного типа в ресурсозатратном функциональном блоке.

Для реализации предложенной стратегии необходимо отслеживать моменты всплесков нагрузки в потоках заявок каждого типа сервиса. Если интервал времени между всплесками меньше среднего время обслуживания заявки соответствующего типа сервиса в ресурсозатратном функциональном блоке, тогда часть заявок последнего всплеска не допускается в систему до тех пор, пока разница между всплесками не превысит среднего значения длительности обслуживания в ресурсозатратной ФБ. Часть заявок, которые задерживаются, определяется как разница между количеством заявок, поступивших во время последнего всплеска и допустимым количеством заявок заданного типа сервиса.

Количество заявок, является допустимым, для заданного типа сервиса находится из задачи линейного программирования, при этом учитывается эфрфективность обслуживания всех типов сервисов при имеющемся объеме ресурсов системы .

\section{III. Выводы}

В докладе представлена стратегия управления входным потоком на сервере оператора мобильной связи , которая бы позволила предотвратить дефицит серверных ресурсов и возникновению задержек в системе .

Продолжение данной работы заключается в создании подходов к управлению обработкой входного потока запросов сервисов конечным пользователем с обратной связью, которые строятся на основе предложенных и включают набор параметров , которые можно настраивать в зависимости от конкретной конфигурации системы , а также набор критериев оценки эффрективности обслуживания потока заявок в целом.

\section{IV. Литература}

[1] Larysa Globa, Mariia Slukysh. Nodal routing with traffic classification// Polish association for knowlage management Series: Studies\&Proceedings №42, 2011, pp 37-46

[2] Пилипенко А.Ю., Чердынцева В.Ф. «Метод уменьшения нагрузки на биллинговую систему в режиме критического состояния» " // Материалы 23-й Международной Крымской конференции "СВЧ-техника и телекоммуникационные технологии” (КрыМиКо'2013). - г. Севастополь. - 2013.

[1] A. Totok, V. Karamcheti. Exploiting Service Usage Information for Optimizing Server Resource Management //ACM Transactions on Internet Technology, Vol. 11, No. 1, Article 1, Publication date: July 2011

[2] Evangelia Kalyvianaki. Resource provisioning for virtualized server applications/ Technical reports published by the University of Cambridge Computer Laboratory are freely available via the Internet: http://www.cl.cam.ac.uk/techreport 
[5] Globa L., Dyadenko A., Reverchuk A. The charging problems in mobile service deployment.// EUROCON 2009 The IEE Region 8 Conference devoted to 150 Anniversary of Alexander Popov, May 19-23, 2009. Saint Petersburg, Russia . 